\title{
Middle-Infrared Observations of DENIS J0255-4700
}

\author{
Michelle Creech-Eakman, Eugene Serabyn \\ Jet Propulsion Laboratory, MS 171-113, 4800 Oak Grove Dr., \\ Pasadena, CA 91109 \\ Glenn S. Orton \\ Jet Propulsion Laboratory, MS 169-237, 4800 Oak Grove Dr., \\ Pasadena, CA 91109
}

Thomas L. Hayward

Gemini Observatory, AURA/Casilla 603, La Serena, Chile

\begin{abstract}
The first infrared photometry of an L-type brown dwarf, DENIS J0255-4700, includes N-band and narrow-band $8.8-\mu \mathrm{m}$ detections, with upper limits in narrow-band 10.3 and $11.7 \mu \mathrm{m}$. Model-independent blackbody fits of existing data yield $\mathrm{T}_{\text {eff }}=1250-1750 \mathrm{~K}$, with models favoring the lower end of that range. Dusty atmospheric models by Allard, Burrows and Marley which match the near-infrared photometry are not completely consistent with our mid-infrared photometry.
\end{abstract}

We report middle-infrared observations of one of the brightest brown dwarfs, the L dwarf DENIS J0255-4700. Estimates of its effective temperature range from $1300 \mathrm{~K}$, from the near-infrared spectral typing (Kirkpatrick et al. 1999), to $1700-1900 \mathrm{~K}$, from analysis of $\mathrm{Cs}$ I and Rb I resonance lines (Basri et al. 2000). The absence of the 670.8-nm Li I line implies that its mass is greater than $50 \mathrm{M}_{\text {Jupiter }}$, with $\sim 60 \mathrm{M}_{\text {Jupiter }}$ more likely (Martin et al. 1999). Apart from the spectroscopy of Martin et al. and Basri et al., the only published observations are in $\mathrm{i}, \mathrm{J}$ and $\mathrm{K}$ bands (Martin et al. 1999, Kirkpatrick et al. 2000).

We used the OSCIR middle-infrared camera/spectrometer in queue time at the Gemini South Observatory on December 6-9, 2001, relying on HD 9362 for primary flux calibration. Our results are summarized in Table 1 and Figure 1. One standard deviation noise values were determined by taking aperture photometry in 4 places on the detector that had no obvious sources. The object was observed to be 5.10 arcsec $\mathrm{E}$ and $5.55 \operatorname{arcsec} \mathrm{S}$ of its DENIS coordinates, a position later confirmed by near-infrared observations at the NASA Infrared Telescope Facility, in February, 2002. We derive a large proper motion of $1.7 \pm 0.2 \mathrm{arcsec} /$ year, consistent with its nearby ( $\sim 5 \mathrm{pc}$ ) distance (Kirkpatrick et al. 2000).

A straightforward blackbody fit to the existing near-infrared and our middleinfrared photometry, weighted by bandwidth, yields an effective temperature of $1500 \pm 250 \mathrm{~K}$. This is probably an overestimate, because near-infrared models 

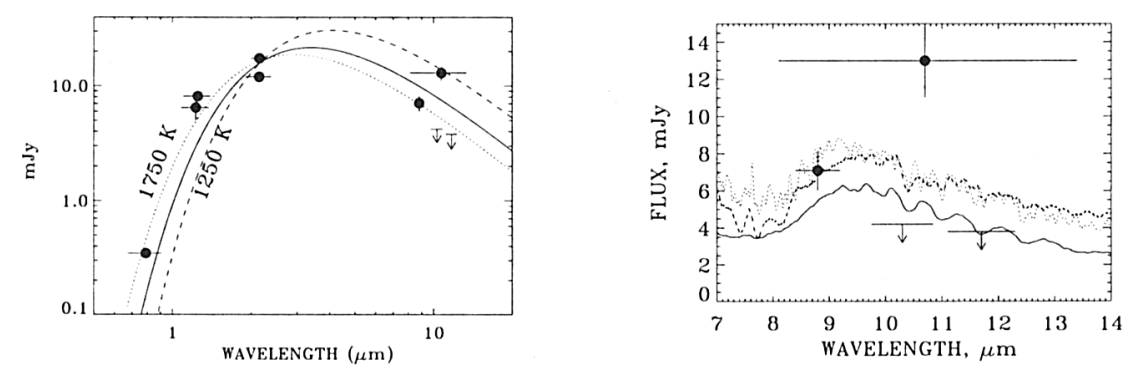

Figure 1. Comparison between observations reported here (Table 1) and (left) best fit of blackbody, together with near-infrared photometry and uncertainties of the fit, (right) partially dusty models by Allard (dashed, this meeting), Burrows et al. (2002, solid), and Marley et al. (2002, dotted) which fit near-infrared photometry.

show that the J- and K-band measurements characterize spectral regions outside substantial gaseous absorptions which reduce the flux.

Table 1. Summary of photometry of DENIS J0255-4700.

\begin{tabular}{cccc}
\hline Filter & Wavelength $(\mu \mathrm{m})$ & Filter Width $(\mu \mathrm{m})$ & Flux $(\mathrm{mJy})$ \\
\hline $\mathrm{N}$ & 10.7 & 5.3 & $13.0 \pm 2.0$ \\
8.8 & 8.8 & 0.8 & $7.1 \pm 1.1$ \\
10.3 & 10.3 & 1.1 & $<4.2(4 \sigma)$ \\
11.7 & 11.7 & 1.2 & $<3.8(4 \sigma)$ \\
\hline
\end{tabular}

Figure 1 compares the data with current "partially dusty" models which fit the near-infrared photometry. The models do not fit the high N-band value. Taken at face value the data would imply that an additional source of emission is needed between 9 and $10 \mu \mathrm{m}$ or longer than $12.2 \mu \mathrm{m}$. On the other hand, the model "continua" could be too low, and the data would be fit best by a brighter continuum that matches the $\mathrm{N}$ band measurement, with an absorption feature required to match the lower flux observed between 8.8 and $11.7 \mu \mathrm{m}$. Clearly further observations are required to corroborate and elucidate our measurements. Spectral observations are highly recommended both in the near and the middle infrared to constrain both dust and compositional properties of DENIS J02554700 .

\section{References}

Allard, F. et al. 2001, ApJ, 556, 337.

Basri, G. et al. 2000, ApJ, 538, 363.

Burrows, A. et al. 2002, ApJ, 573, 304.

Kirkpatrick, A. D. et al. 2000, AJ, 120, 447.

Marley, M. et al. 2002, ApJ, 568, 335.

Martin, E. et al. 1999, AJ, 118, 2466. 